\title{
Discussion on the Tendency of Flat UI Design for Mobile Terminals
}

\author{
Jian Zhang \\ Sichuan Fine Arts Institute \\ Chongqing, China 401331
}

\begin{abstract}
The characteristics of flat UI design for mobile terminals, compared with quasi-physical design style, are introduced. The influence and significance of these design styles on interaction are discussed in this paper. The driving factors of flat design which is prevalent in mobile terminals are also analyzed. Based on these discussions, the application and value of flat UI design in mobile devices from the point of users' views and the future developing tendency are be studied in this paper.
\end{abstract}

Keywords-interface; interactive; flat design; quasi-physical design; User-Experience

\section{INTRODUCTION}

With the release of iOS7 in June 2013 that is the latest mobile system of Apple, it became the focus once again that design style of flat UI which made the whole system more concise without gorgeous, which is the opposite perfection of quasi-physical design that is favored by Apple. Previously, Microsoft maximized flat UI design with Windows UI which came from the Swiss International Typographic Style with the characteristics of neat, rigorous, rational, and meticulous, accurate [1]. With the rise of mobile Internet, the flat UI design style came back again. Systems, web sites or software designers seem to be eager to abandon quasi-physical design and meet the era of flat UI [2]. In essence, that is not only a change of visual style, but also a change of design language and thinking.

\section{QUASI-PHYSICAL UI DESIGN}

\section{A. Interaction of Quasi-physical UI Design}

Quasi-physical is the imitation for entity in interface, which makes the digital objects more close to real. It is conducive to improve User-Experience according to official HMI Guidelines of Apple which presents that "the more close to entity the interface and behavior are, the easier they are understand by users and the easier they are used".

The quasi-physical design of interface includes a simulation of express of the real world (such as texture, texture, sound, etc.), and interaction method between the interface and people (which is often ignored). When simulating express of the real word, quasi-physical design can take an intuitive and efficient express, and make interface beautiful, and create a gorgeous visual effect to

The project of "Research on graphic language of Internet mobile terminal interface" from Chongqing Municipal Education Committee, with project number of KJ1400801. meet more users' needs of entertainment and emotion [3]. Prior to IOS, quasi-physical design was often used in game interface in order to create a sense of immersion. In game interface, there are many parchments, rocks, metal texture and quality, even decorated by architecture, sculpture, or dermatoglyphic pattern in accordance with the decade and style of game scenes, which is a restore of the real word and a improvement of artistic effect, is conducive to create immersion sense and pleasure, as shown in "Fig. 1".

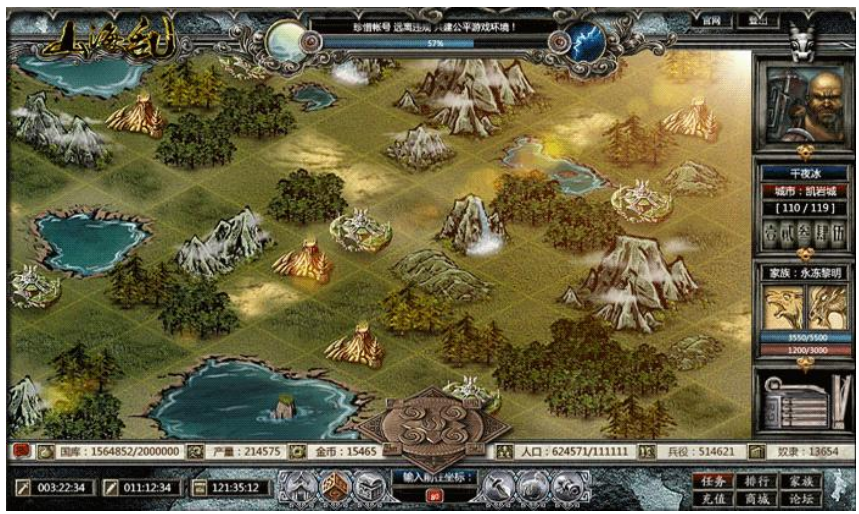

Fig. 1. The game interface of "Shanhailuan"

\section{B. Limitation of Quasi-physical Design}

Quasi-physical design that is successfully used in game interface can't be used in office interface or other serious interface, such as stock trading software interface. Secondly, it needs to face the difference between virtual interface and real entities. Before realization of universal computing, at present, as a carrier, the screen can't overcome dimensions differences, which leads to a failure of imitating real world. When a program interface almost completely imitates real entities, there is a corresponding behavior method between them to be expected by users. But in some cases, this design only meets visual senses rather than functions, which causes "only shape and non-sprit". In addition, facing different users with different habits and experience, and regional and cultural differences between the user groups, a interface design style can't be fully suitable for all users. Therefore, quasi-physical UI design should convert real entities to screen interface language, in order to respect the existing habits and mental model of users in visual, and use corresponding gesture in operation. 
In general, the deficiency of quasi interface is that it can influence instinctive level in the user emotional experience because attracting and satisfying the user in the vision. However, interactive function itself is more unlikely to be improved. With the popularization of personal computer, a decreasing number of people need these visual cues to understand the function of a button or icon.

\section{FLAT DESIGN OF UI}

\section{A. Less Is More}

In terms of vision and communication, "less is more", which is the architect Mies van De Luoti out in the design of simple principle, can prove the rationality of the UI flat. A main reason is that his statement is the formed-simple, and rational design with high function, as opposed to the decoration design style [4]. The chief designer, Jonathan - AI, coming from Apple Inc, declares the minimalist design philosophy: "the beauty of simplicity will be far-reaching, inclusive and efficient. True minimalism not only abandons a redundant modification, but also brings order to complex." Flat design can be understood best - minimalist, which means that using the light weight and simple design communicates the key information, guiding by the visual focus allows users to quickly complete the operation.

\section{B. Design Concept of Information as the Main Body}

Give up any additional effect, highlight important information and simplify the process of interaction. Visual level, UI flat design can be understood popularly: without three dimensional attributes, such as texture, projection, slant, feather edges, and other relief effects. In essence, the core of UI flat design is to emphasize the information itself, rather than the redundancy of the interface elements. For example, the inspiration of Windows UI came from transport system which is demanded to help people quickly to find the information they need in a short time. The objective of flat interface is not only to create visual stimulation, but to solve interferences and retain the information concerned by users, show simple and readable content by alignment, let users immerse in their favorite content and pay attention to core content. Reducing visual decoration of concept and interface level, it uses more interaction which is mastered by users.

\section{Limitation of Flat UI Design}

As said by some people that "Flat age is a good age for commonplace designers", undoubtedly it had been understood flat in essence, like any design trends, would inevitably be abused without thinking by some people who drifts with the tide. In addition, as Swiss international style, flat UI design also has the characteristics of indifference, rationality and functionalism. Flat UI design is undoubtedly a double-edged sword, too rational and formulation will lead to loss of individuality and ignore emotional needs of users, fails to care for the influence of personal aesthetic and traditional on people; lack of sensibility and humanistic will lead to a great limitation to designers. It emphasizes understandable and memorable which need the characteristics of simple form, anti-decoration, emphasizing function, high rationality and systematic, such as maximizing abstraction of texture in interface design, abandoning all basic elements express space depth, dimension and light, separating the connection of virtual cognition and real world, increasing cognitive cost, especially children and users lack of Internet experience.

\section{INFLUENCE OF MOBILE INTERNET ON UI STYLE}

As shown in " Trend report of mobile Internet in 2013" issued by Baidu, PC Internet is increasingly migrating to mobile terminal; the per capita time of using mobile Internet has exceeded PC Internet and keeps a rapid growth, up to March 2013, the gap between them has been expanded to $29 \%$, mobile phones have been replacing PC and become the most common terminal.

\section{A. Simple, Efficient and Universal Interface}

In the software interface, it will mislead the users that only imitating physical form rather than pointing original function. Such as the frame of a calendar software that imitates tear edge of paper or the metal buckles of a telephone book in margin, which are easy to misunderstand or disturb users, even the concept is accurate will convey different effects. As shown in "Fig. 2" the comparison of two icons, from point of user experience, according to the interpretation process of metaphor in "Fig. 3", analyze effect of transferring information. The common interpretation information include "contact person" (shown as a avatar directly, has more strongly visual signal in iOS7), "sort and group information "(shown in the form of letters and tabs, almost impossible to identify in iOS6). The more information in iOS6 than iOS7, including "binding ring ", " metallic "," shadow "," yogon kraft paper "," hollow "and other visual information, and the implied emotional information including" nostalgia "," smooth "and so on, may increase the burden of information interpretation for users.
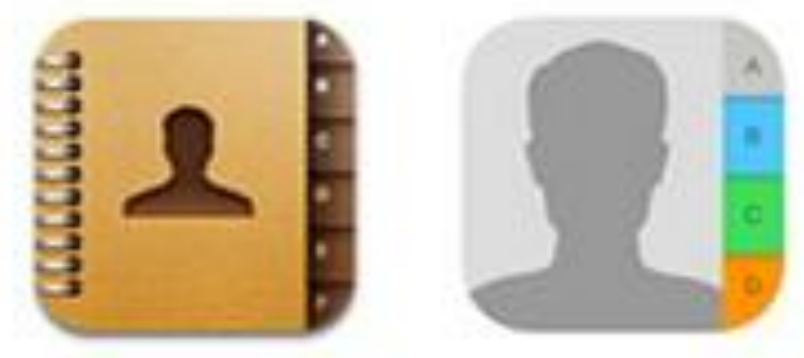

Fig. 2. Contact books of iOS6 (left) and iOS7 


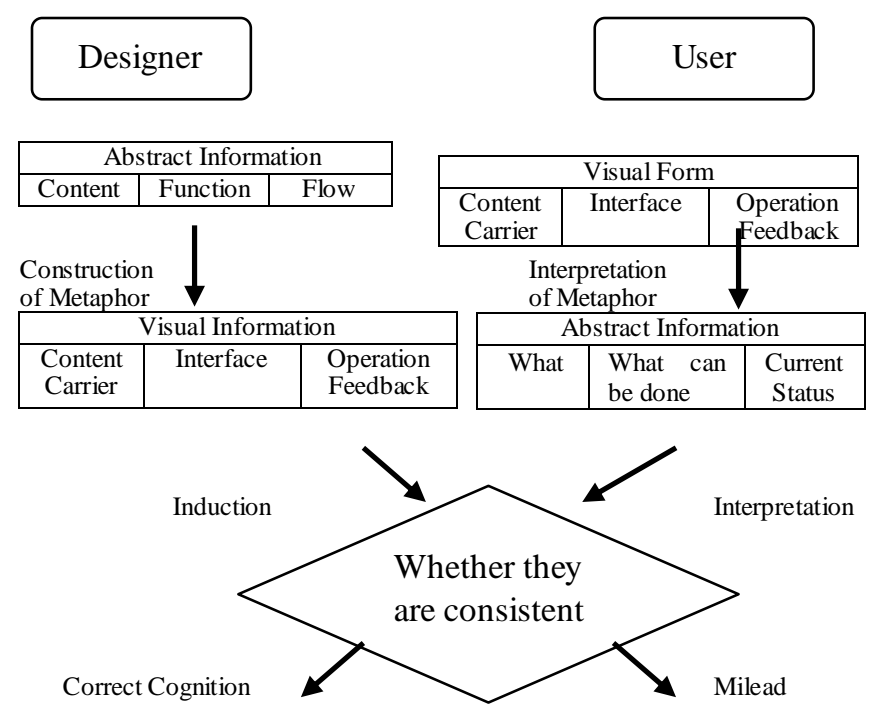

Fig. 3. Encoding and decoding flow of interface design

Flat design on the small screen can obtain a better performance, especially in the mobile terminal. With the development of the mobile Internet, the dissemination of information in the speed and scale has reached an unprecedented level, which results in global information sharing and interacting. However, a large amount of information brought by this often makes people confused. As the interface of the system platform and application software of mobile terminal, its complexity has overtaken that of PC. Compression of the displayed space makes mobile terminal compact and communication convenient, and meanwhile brings more challenges on the interface design. Collecting information effectively and delivering communication accurately has become particularly important. The rapid growth of information and services provided by the mobile terminal, in order to guide the interface form program icon, has been unable to satisfy so much of the information needs. In ios6 main interface, we cannot acquire the message about the contents and only know the number of unread messages, gaining preview by going into the mailbox from program icon. On the other hand, the main message of the mail in Windows UI will directly present in the message area, and users can obtain more information as a animated scrolling form in this level, such as the application of weather or other parts, as shown in "Fig. 4". Therefore, the interface design need to present information directly-- the content is the interface. When the information content with true colors becomes the main interface, purely decorative design in the past began to be a burden on the content of the interface, which need deliver focus of consciousness for a large number of information content. As a result this design inevitably have been weakened and peeled gradually. For example, IOS 7 interface in ios6 basis has removed the redundancy of the color, size, and texture elements, which highlights subject information and also displays information in more levels. Windows UI is a relatively extreme example, and from "Fig. 4", we can see the focus of its interface design, returning to the information, color distribution and dynamic metaphors, etc. For consistency in the interface, IOS by way of a single full program running avoids the strong contrast, caused by the application of different visual style. By contrast, flat design makes the relationship of functional interface is more visual consistency and coherence, and allows them to be together in the main interface shown the content and information. In addition, the interface design not only requires simple, efficient style, but also need to use international general visual language, as the increase of international communication and international trade has promoted the formation of internationalism design style. That the interface is flat suits the developing of era.
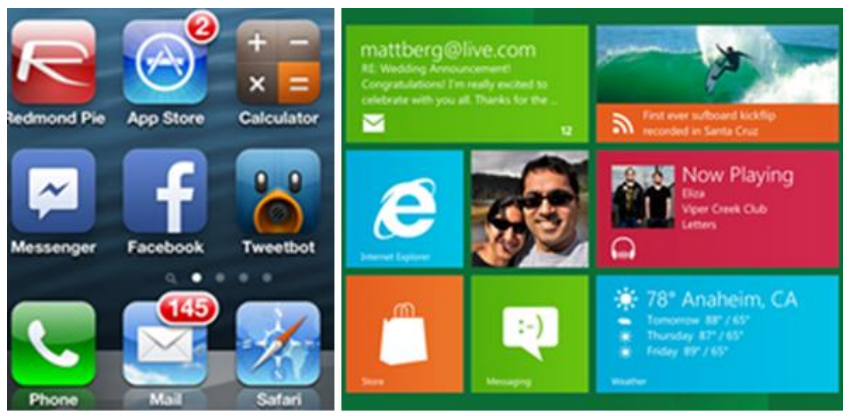

Fig. 4. Interfaces of iOS6(left) and Windows UI(right)

\section{B. Requirement of Response Type Design}

With the development of mobile Internet, this also brings a problem that how we can set up a website, which is suitable for all user access. That means cross platform and multi screen adaptation. In the past, a mobile website need to be built for mobile users, and now the solution of the program is response type design. Compared with this, flat interface is easier to implement responsive design, because quasi static images of objects cannot adapt to the response requirements. This is why many designers began to avoid paying attention to texture designed and turn into very simplified design. A web site is built, focusing on the dynamic layout of different browsers and devices, which is called responsive design. It can dynamically change the layout and content of the page based on the size of the browser and device, seen "Fig. 5". June 2013, the revised video site Youku uses a flat design style, to achieve interactive experience among $\mathrm{PC}$, mobile phone and tablet style and content.

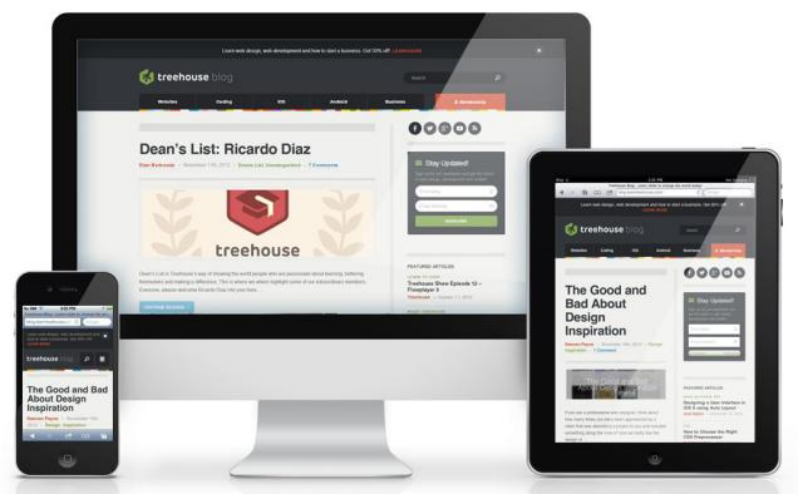

Fig. 5. Responsive Design for Website 


\section{Flat UI Design in Point of New Technology Popularizing}

Nowadays, the biggest change brought by the mobile Internet is that the virtual world is combined with the real world. Improving the reality skills on the mobile Internet to apply is a trend in the future. The application form is mainly reflected to enhance the real information, which means that information on the Internet additionally displays in real information. Whether it is "the sixth sense" equipment, OmniTouch in form of projection display interface or the Google glasses augmented reality display is in the physical environment to show interface of mobile terminal. This requires that interface can adapt different color, light, texture and different surface display, and flat interface style than the quasi physical styles are more likely to do this. In addition, this style can reduce the interference of redundant information, and give the main information. At the same time, more the interaction process is further simplified, namely, the operation level of the flat, as shown in "Fig. 6". Therefore, as the above devices are presented in the interface style, in the present or in a short period of time, the mobile device interface will be flat to occupy the mainstream. For instance, Microsoft's vision of a series of video also indicates this direction.
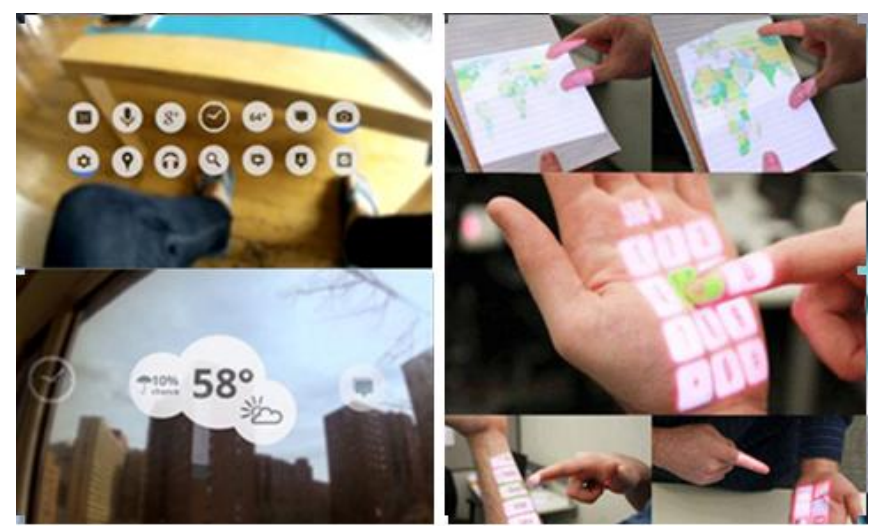

Fig. 6. Google Glass UI ( left) and OmniTouch UI (right)

\section{CONCLUSION: INTERSECTION AND INTERGROWTH OF THE TWO DESIGN STYLES}

The user's aesthetic, visual experience, interactive experience is different. Which design style can be chosen depends on whether it can meet the needs of users at present. The pursuit of the two design style is to allow users to interact more efficiently and more comfortably, which is thought not only from the angle of the function and technology of rational, but also from emotional care level, giving the product a more human nature and aesthetics. The opinion in design process alternately repeats, and designers need to find equilibrium point, namely, two kinds of design style of the intersection.

The dramatic development of interactive technology gives a great extension of human ability. UI designers are always exploring and challenging new design styles, and are keen to see the changing and progress. Flat and quasi physical given or abandoned, cannot be generalized and need to take the user as the center. Furthermore, goal oriented, different user groups, different types of hardware, different functional demands and emotional appeal decide interface structure and form. Therefore, regardless of the flat or materialized design style, interface constantly focus on the user's needs, and take the user as a starting point design, which can build a comfortable, convenient and easy efficient interface to use. If taking into account the needs of users at the same time, we also can meet the aesthetic pursuit, helping between the product and the user to establish a stable and harmonious relationship. This really makes science to serve the people.

\section{REFERENCES}

[1] WangShouZhi. History of Modern Design(2th ed)[M]. ShenZhen: New Century Publishing House,2001.

[2] Carrie Cousins[ US]. Flat Design: Can You Benefit from the Trend? [EB/OL] : http://designmodo.com/flat-design/

[3] Donald · A $\cdot$ Norman[US]. translate by FuQiuFang,ChengJinSan. Emotional Design (3th ed)[M].BeiJing : Publishing House of Electronics Industry,2006.

[4] Sacha Greif[ US]. The battle between flat design \& Skeuomorphism flat pixels. [EB/OL]:http://sachagreif.com/flat-pixels. 